\title{
Mycobacterium avium complex disease: prognostic implication of high-resolution computed tomography findings
}

\author{
S. Kuroishi*, Y. Nakamura*, H. Hayakawa" ${ }^{\#}$ M. Shirai" ${ }^{\#}$, Y. Nakano", K. Yasuda ${ }^{+}$, \\ T. Suda*, H. Nakamura* and K. Chida*
}

ABSTRACT: To evaluate the prognostic implications of computed tomography (CT) findings in assessing responses to treatment in Mycobacterium avium complex (MAC) pulmonary disease without underlying lung disease, high-resolution (HR)CT findings were correlated based on the results of sputum conversion after anti-MAC therapy.

A total of 59 patients underwent HRCT before treatment and the therapeutic efficacy was evaluated by the results of sputum conversion.

Atelectasis, cavities and pleural thickening on HRCT were significantly more frequent and extensive among patients in the sputum nonconverted group than among those in the converted group. Furthermore, bronchiectasis was also significantly more extensive among patients in the nonconverted group, even though there was no significant difference in frequency between these two groups.

These results suggest that high-resolution computed tomography findings are good predictors of response to treatment in Mycobacterium avium complex pulmonary disease.

KEYWORDS: High-resolution computed tomography, Mycobacterium avium complex, nontuberculous Mycobacterium, prognosis, sputum conversion

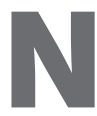

ontuberculous mycobacteria (NTM), especially Mycobacterium avium complex (MAC), are being recognised with increasing frequency as clinical pathogens of chronic lung disease in immunocompetent patients [1]. These infections have traditionally been difficult and frustrating to treat. Although there are some reports demonstrating the efficacy of clarithromycin (CAM)-containing regimens, current drugs are not as effective as they are in treating pulmonary tuberculosis [2-5].

Several investigators have reported radiological findings of patients with MAC pulmonary disease. MoOre [6] reviewed the computed tomography (CT) and high-resolution (HR)CT findings of patients who had cultures positive for NTM, finding that bronchiectasis was one of the most common findings in combination with nodules. SWENSEN et al. [7] found that CT findings of small lung nodules in association with bronchiectasis had a high level of sensitivity, specificity and accuracy in predicting positive cultures for MAC. Therefore, HRCT findings are useful for making a diagnosis of MAC pulmonary disease. However, there are few reports describing $\mathrm{CT}$ findings in relation to therapeutic outcome in MAC pulmonary disease. The present study assessed the prognostic implication of HRCT findings based on the results of sputum conversion after anti-MAC treatment. Furthermore, changes in CT findings on follow-up examinations were also evaluated.

\section{MATERIALS AND METHODS}

\section{Patient selection}

The medical records of patients who received an initial diagnosis of MAC in the present authors' facilities (Hamamatsu University School of Medicine, National Hospital Organization Tenryu Hospital, Seirei Mikatahara General Hospital and Iwata Municipal Hospital; all Shizuoka, Japan) from August 1995 to December 2001 were reviewed. All patients fulfilled the American Thoracic Society (ATS) criteria for the diagnosis of NTM infection [8].

In the present study, patients who were basically immunocompetent or who had other lung diseases, such as chronic obstructive pulmonary disease, interstitial lung disease, lung cancer or healed tuberculosis, were excluded. Patients who had a history of treatment for MAC infection or pulmonary surgery were also excluded. All
AFFILIATIONS

*Second Division, Dept of Internal Medicine, Hamamatsu University School of Medicine,

"Dept of Respiratory Medicine,

National Hospital Organization Tenryu Hospital,

"Dept of Respiratory Medicine, Seirei Mikatahara General Hospital, and

${ }^{+}$Dept of Respiratory Medicine, Iwata Municipal Hospital, Shizuoka, Japan.

CORRESPONDENCE

Y. Nakamura

Second Division

Dept of Internal Medicine

Hamamatsu University School of

Medicine

Handayama 1-20-1

Hamamatsu

Shizuoka

431-3192

Japan

Fax: 81534352354

E-mail: nakayuta@hama-med.ac.jp

Received

June 202007

Accepted after revision:

February 192008

STATEMENT OF INTEREST

None declared.

European Respiratory Journal Print ISSN 0903-1936 Online ISSN 1399-3003 
patients were treated with a CAM-containing regimen for at least 12 months. Patients who discontinued therapy because of adverse effects were excluded.

\section{Data collection}

Clinical data, including causative organism of MAC infection, sex, age, performance status, body mass index, laboratory data (white blood cell count, erythrocyte sedimentation rate, total protein, albumin, C-reactive protein, arterial oxygen tension), and dose of CAM were obtained from the patient's medical records.

\section{Specimen preparation}

At least three adequate sputum cultures were obtained from all patients. Specimens stained with Ziel-Neelsen and Gram's stains were reviewed from all cases and cultured for mycobacteria with other bacteria and fungi. Mycobacteria were cultured with 3\% Ogawa egg medium (Nissui Pharmaceutical, Tokyo, Japan). Identification of Mycobacterium avium and Mycobacterium intracellulare was made using the Cobas Amplicor Mycobacterium test (Roche Diagnostics Japan, Tokyo, Japan). All patients were diagnosed from sputum specimens.

\section{CT scans}

CT scans were performed with X-Vigor (single-slice helical CT; Toshiba, Tokyo, Japan), X-vision (single-slice helical CT; Toshiba), HiSpeed Advantage (single-slice helical CT; GE Yokokawa Medical Systems, Tokyo, Japan), or LightSpeed QX/i (multi-slice helical CT; GE Yokokawa Medical Systems) scanners. The radiographic factors were as follows: X-Vigor (2$\mathrm{mm}$ slice thickness, 20-mm gaps), X-Vision (2-mm slice thickness, 10-mm gaps), HiSpeed Advantage (3-mm slice thickness, 10-mm gaps), and LightSpeed QX/i (1.25-mm $\times 4$ collimation, 2.5-mm slice thickness, $15-\mathrm{mm}$ gaps). Image data of HRCT were reconstructed with $512 \times 512$ matrix and 20$25 \mathrm{~cm}$ field of view using a high-resolution algorithm. The presence of small nodule $(\leqslant 5 \mathrm{~mm})$, nodule $(>5 \mathrm{~mm})$, mass $(>3 \mathrm{~cm})$, bronchiectasis, air space consolidation, atelectasis (lobar atelectasis and segmental atelectasis), cavity formation, hilar or mediastinal nodes $>1 \mathrm{~cm}$ in diameter and pleural thickening was evaluated in each lung lobe (fig. 1). In all patients, the number of lung lobes that contained lesions was counted (right upper lobe, right middle lobe, right lower lobe, left upper lobe, lingula, left lower lobe). The finding of bronchiolectasis was also included in bronchiectasis.

In 23 patients, follow-up CT scans were obtained 1247 months (mean 25 months) after the initial scan. The CT findings on the follow-up scan were compared with those on the initial CT scan. In this follow-up CT scan, the improvement of CT findings was assessed, which was defined as disappearance or decrease of each finding. The initial and follow-up CT scan findings were reviewed by two observers (M. Shirai and Y. Nakamura), who had no knowledge of the patients' clinical data, and consensus was obtained.

\section{Treatment}

All patients were initially treated with a CAM-containing regimen according to the recommendation by the ATS [8]. All patients were treated with rifampicin $\left(0.3-0.45 \mathrm{~g} \cdot\right.$ day $\left.^{-1}\right)$, ethambutol $\left(0.5-0.75 \mathrm{~g} \cdot \mathrm{day}^{-1}\right)$, and CAM $\left(0.4-0.8 \mathrm{~g} \cdot \mathrm{day}^{-1}\right)$ with or without the addition of streptomycin (SM) for the first 2 months (0.4-0.6 g three times weekly). These regimens were subsequently modified individually due to adverse reactions. Treatment was continued for $\geqslant 12$ months.

\section{Estimation of outcome}

Sputum conversion was defined as consecutive negative sputum cultures over a 3-month period, with the time of conversion defined as the date of the first negative culture. When the patient's condition improved and excretion of sputum disappeared completely, sputum induction with sterilised $3 \% \mathrm{NaCl}$ was performed. If the patient could not expectorate sputum even after the sputum induction, it was considered that sputum had converted to negative. Eventually, all patients were divided into two groups. The first group consisted of the patients who converted their sputum to negative (converted group). The other group consisted of the patients who could not convert their sputum to negative (nonconverted group).
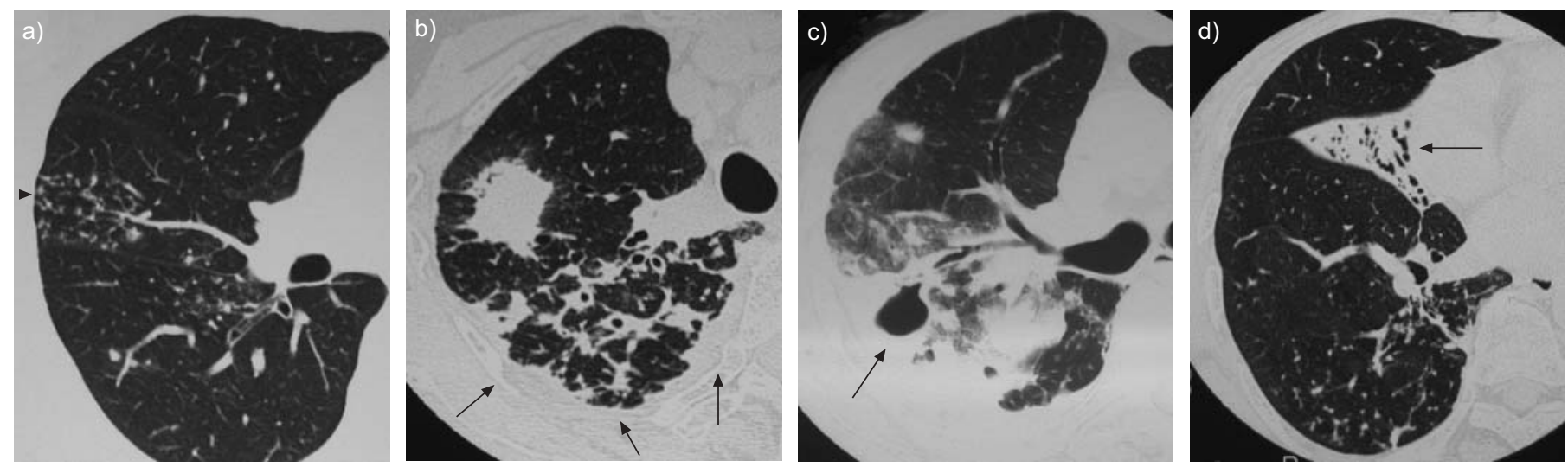

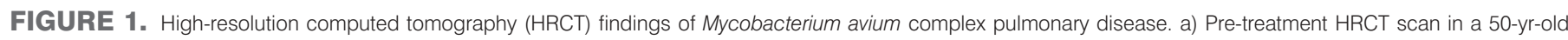

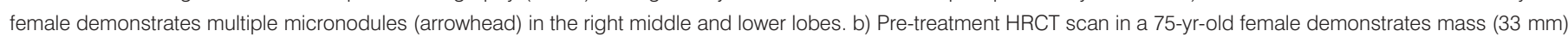

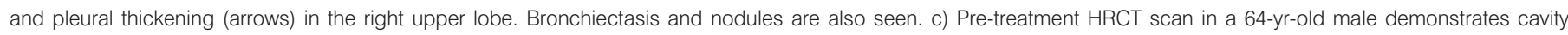

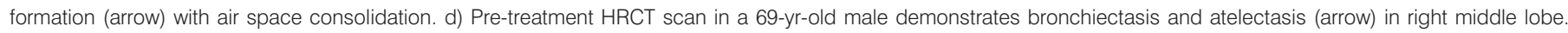
Multiple micronodules are also seen in the right lower lobe. 
TABLE 1 Characteristics of patients with Mycobacterium avium complex pulmonary disease

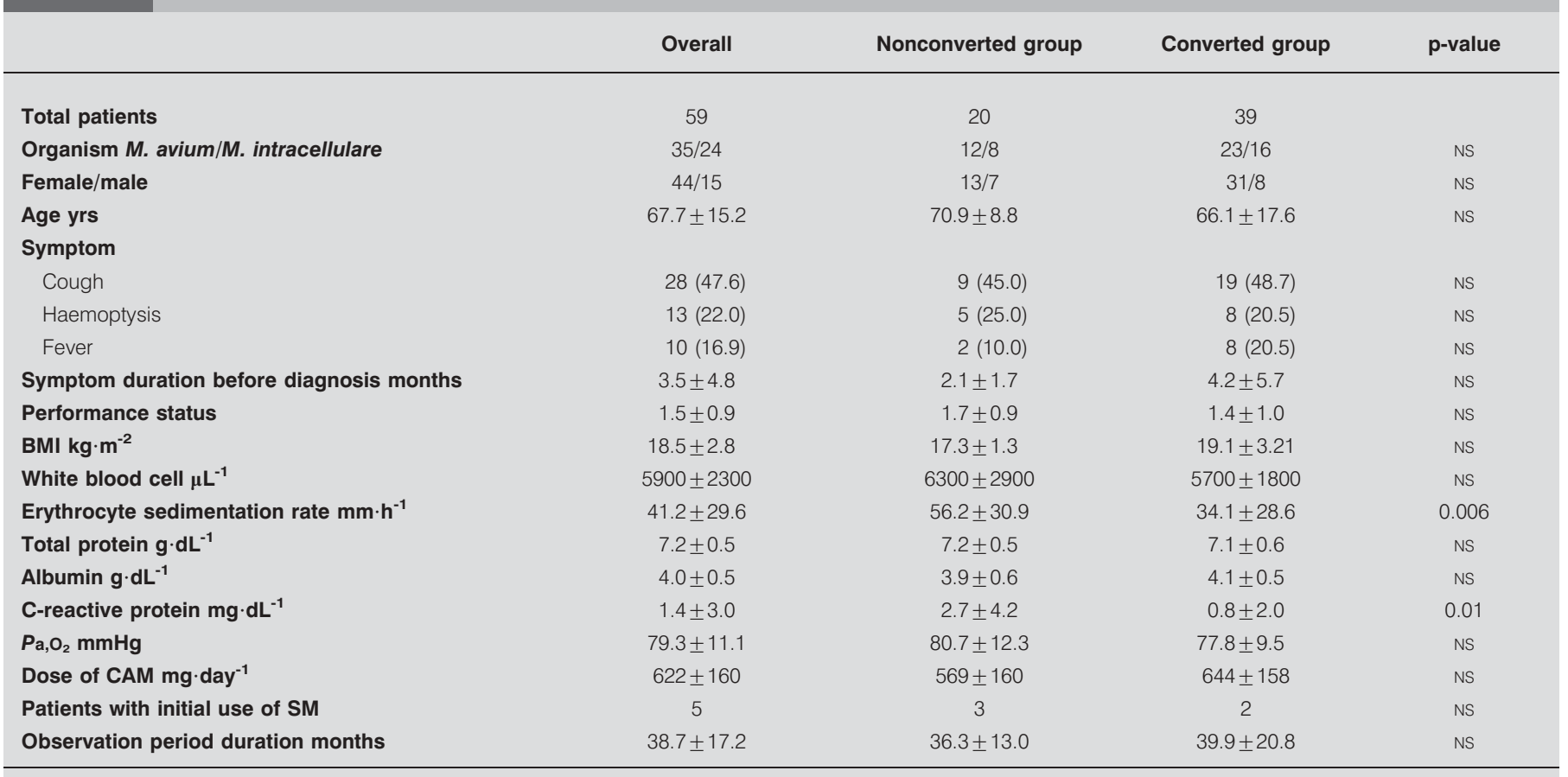

Data are presented as $n$, mean \pm SD or $n(\%)$. The $p$-values shown are for the nonconverted versus converted group. BMl: body mass index; $P a, O_{2}:$ arterial oxygen tension; CAM: clarithromycin; SM: streptomycin; NS: nonsignificant.

\section{Statistical analysis}

Values are expressed as the mean \pm SD. For the statistical evaluation of differences between the two groups, unpaired t-test and the Mann-Whitney U-test were used for parametric data and nonparametric data, respectively. Fisher's direct way was used for comparison between the two groups. Values of $p<0.05$ were considered to be statistically significant.

\section{RESULTS}

\section{Patients and disease}

The total number of patients who satisfied the ATS criteria in the current authors' institutions during the study period was 190. A total of 72 patients were excluded because of underlying lung disease (60 patients) or previous history of treatment for MAC lung disease (12 patients). Another 45 patients were excluded because they were not examined at the initial CT scan. A further 14 patients were withdrawn from the study because one of the following occurred: a request for withdrawal from the treatment was made by the patient; the patient was lost to follow-up; or adverse events occurred that required the discontinuation of treatment. In total, 54 patients had a positive sputum sample on smear with at least two positive sputum samples on culture. The remaining five patients had at least three positive sputum samples on culture with a negative sputum sample on smear. The mean time to sputum conversion was $10.5 \pm 9.0$ months in the sputum converted group.

\section{Clinical characteristics}

The clinical characteristics of patients with MAC pulmonary disease are summarised in table 1 . Although $74 \%$ were female, there was no difference in sex between the sputum converted and the nonconverted groups. Patients in the nonconverted group had a significantly higher erythrocyte sedimentation rate $(p=0.006)$, and higher serum level of $C$-reactive protein $(p=0.01)$. No significant difference in the dose of CAM per day was observed between the sputum nonconverted group and the converted group. In addition, only five patients initially received SM; however, there was no significant difference in the result of sputum conversion.

\section{HRCT findings}

Table 2 outlines the HRCT findings of the patients in the nonconverted and converted groups. Atelectasis, cavities and pleural thickening in HRCT findings were significantly more frequent among patients in the nonconverted group than those in the converted group $(p=0.03, p=0.02$ and $p<0.0001$, respectively). Although a few combinations of more than one finding on HRCT were also significantly different in the nonconverted group compared with the converted group (all combinations including at least one HRCT finding that was significantly more frequent in the nonconverted group by itself; table 2), no combination of findings could predict with certainty conversion in the individual patient.

Following this, the extent of the HRCT findings was compared between the two groups. The findings related to the number of lobes involved: bronchiectasis, as well as atelectasis, cavities and pleural thickening were significantly more extensive in patients in the nonconverted group than in those in the converted group (table 3).

To investigate the improvements in HRCT findings, the changes in HRCT findings between the initial and follow-up 


\begin{tabular}{|c|c|c|c|c|}
\hline \multirow[t]{2}{*}{ TABLE 2} & \multicolumn{4}{|c|}{$\begin{array}{l}\text { Frequencies of high-resolution computed } \\
\text { tomography findings in patients with } \\
\text { Mycobacterium avium complex pulmonary } \\
\text { disease }\end{array}$} \\
\hline & Overall & $\begin{array}{l}\text { Nonconverted } \\
\text { group }\end{array}$ & $\begin{array}{l}\text { Converted } \\
\text { group }\end{array}$ & p-value \\
\hline Patients & 59 & 20 & 39 & \\
\hline Micronodules & $59(100)$ & $20(100)$ & $39(100)$ & NS \\
\hline Nodules & $48(81.3)$ & $15(75)$ & 32 (82.1) & NS \\
\hline Mass & 5 (8.5) & $2(10)$ & $3(7.7)$ & NS \\
\hline Consolidation & $13(22.0)$ & $6(30)$ & 7 (17.9) & NS \\
\hline Bronchiectasis & 45 (76.2) & $18(90)$ & 27 (69.2) & NS \\
\hline Atelectasis & 35 (59.3) & $16(80)$ & $19(48.7)$ & 0.03 \\
\hline Cavity formation & $22(37.3)$ & $12(70)$ & $10(25.6)$ & 0.02 \\
\hline Pleural thickening & $16(27.1)$ & $12(70)$ & $4(10.2)$ & $<0.0001$ \\
\hline Pleural effusion & $5(8.5)$ & $2(10)$ & $3(7.7)$ & NS \\
\hline Lymphadenopathy & $21(35.6)$ & $8(40)$ & 13 (33.3) & NS \\
\hline
\end{tabular}

Data are presented as $n$ or $n(\%)$. The $p$-values shown are for the nonconverted versus converted group. NS: nonsignificant.

examination were assessed in the available cases. There were no significant differences in improvements in HRCT findings between the nonconverted and converted groups. However, in all available cases, improvements of micronodules and nodules were higher than those of bronchiectasis and pleural thickening on follow-up examinations (table 4).

\section{DISCUSSION}

Previously, it has not been clear that CT findings could predict the therapeutic outcome in patients with MAC pulmonary disease. TANAKA et al. [2] have shown that the extent of the disease on chest radiography did not correlate with the results of sputum conversion in MAC patients treated with CAMcontaining regimens. In contrast, KOBASHI and MATSUSHIMA [3] reported that sputum conversion rates were significantly poorer in patients with advanced disease throughout the unilateral lung field. In the present study, a consecutive group of MAC patients was reviewed, and CT findings were examined in relation to the results of sputum conversion. It was found that atelectasis, cavities and pleural thickening on HRCT were significantly more frequent findings in patients in the therapeutically poor response group. Furthermore, those findings among patients in the therapeutically poor response group were significantly more extensive than those in patients with a therapeutically good response. Additionally, bronchiectasis was also significantly more extensive in patients in the therapeutically poor response group. These results suggest that HRCT findings might be good predictors of response to treatment in MAC pulmonary disease.

Several investigators have reported CT findings of NTM patients without underlying lung disease [6, 7, 9-15]. In these communications, micronodules (95-100\%), bronchiectasis (65$94 \%$ ), cavity formation (13-43\%), air space consolidation (26$62 \%)$ and pleural thickening (17-52\%) were frequently observed. Consistent with these reports, in the present study, micronodules $(100 \%)$ and bronchiectasis $(76 \%)$ were the most

\begin{tabular}{|c|c|c|c|c|}
\hline \multirow[t]{2}{*}{ TABLE 3} & \multicolumn{4}{|c|}{$\begin{array}{l}\text { The numbers of lung lobes involved in disease in } \\
\text { oatients with Mycobacterium avium complex } \\
\text { oulmonary disease }\end{array}$} \\
\hline & Overall & $\begin{array}{c}\text { Nonconverted } \\
\text { group }\end{array}$ & $\begin{array}{c}\text { Converted } \\
\text { group }\end{array}$ & p-value \\
\hline Patients & 59 & 20 & 39 & \\
\hline Micronodules & $3.8 \pm 1.6$ & $4.3 \pm 1.4$ & $3.6 \pm 1.8$ & NS \\
\hline Nodules & $1.4 \pm 1.0$ & $1.1 \pm 0.8$ & $1.6 \pm 1.2$ & NS \\
\hline Mass & $0.1 \pm 0.2$ & $0.1 \pm 0.2$ & $0.1 \pm 0.1$ & NS \\
\hline Consolidation & $0.5 \pm 1.2$ & $0.8 \pm 1.5$ & $0.4 \pm 1.0$ & NS \\
\hline Bronchiectasis & $2.1 \pm 1.5$ & $2.9 \pm 1.8$ & $1.7 \pm 1.4$ & 0.006 \\
\hline Atelectasis & $1.3 \pm 1.3$ & $1.9 \pm 1.4$ & $1.0 \pm 1.3$ & 0.02 \\
\hline Cavity formation & $0.5 \pm 0.8$ & $1.0 \pm 1.1$ & $0.3 \pm 0.7$ & 0.02 \\
\hline Pleural thickening & $0.6 \pm 1.1$ & $1.2 \pm 1.1$ & $0.3 \pm 1.0$ & 0.0002 \\
\hline
\end{tabular}

frequent findings, followed by cavity formation $(37 \%)$, air space consolidation (22\%) and pleural thickening (27\%).

Intriguingly, bronchiectasis was significantly more extensive in the sputum nonconverted group than in the converted group in the present study, even though there was no significant difference in frequency between these two groups. FujitA et al. [16] demonstrated on pathology that peribronchiolar lesions, including bronchiolitis and bronchiectasis with granuloma formation, were caused by MAC infection. These findings suggest that extensive bronchial lesions represent the progression of NTM disease, while $\mathrm{KOH}$ et al. [17] have reported that CT findings of bronchiectasis involving more than five lobes are highly suggestive of NTM pulmonary disease in patients with bilateral bronchiectasis and bronchiolitis in chest CT. Collectively, extensive bronchiectasis might be an important diagnostic indicator as well as having prognostic implications for NTM.

There are some reports describing changes in CT findings with therapy in NTM patients. ОвАҮASH et al. [18] reported that the finding of bronchiectasis worsened in $\sim 2$ yrs in a follow-up study; however, centrilobular nodules did not deteriorate. KIM et al. [10] reported that improvement of cavitary lesion (24\%) and bronchiectasis (32\%) was much less than that of nodular shadow (51\%). Furthermore, in a separate study by a different group, KIM et al. [11] have suggested that bronchial nodules evolve into focal bronchiectasis. In the present study, consistent with these studies, the findings of pleural thickening or bronchiectasis did not improve. Conversely, micronodules or nodules improved in $70 \%$ of cases. These results suggest that each finding evolved separately. Furthermore, these results indicate small nodules are reversible in response to drug therapy. In contrast, once bronchiectasis is completed, there is little prospect for radiographic improvement.

Patients in the sputum nonconverted group in the present study had extensive lesions, including "irreversible" bronchiectasis in CT findings. In addition, the levels of C-reactive protein or erythrocyte sedimentation rate in patients in the nonconverted group were significantly higher than those in the converted group. These findings indicate that patients in the 


\begin{tabular}{lcccc}
\hline TABLE 4 & $\begin{array}{c}\text { Improvements of high-resolution computed } \\
\text { tomography findings on follow-up examinations }\end{array}$ \\
& Overall & $\begin{array}{c}\text { Nonconverted } \\
\text { group }\end{array}$ & $\begin{array}{c}\text { Converted } \\
\text { group }\end{array}$ & p-value \\
& & & & \\
& & & & \\
\hline Micronodules & $65(15 / 23)$ & $(3 / 7)$ & $75(12 / 16)$ & NS \\
Nodules & $60(12 / 20)$ & $20(1 / 5)$ & $73(11 / 15)$ & NS \\
Mass & $67(2 / 3)$ & $0(0 / 0)$ & $67(2 / 3)$ & \\
Consolidation & $71(5 / 7)$ & $33(1 / 3)$ & $80(4 / 5)$ & NS \\
Bronchiectasis & $5(1 / 19)$ & $14(1 / 7)$ & $0(0 / 12)$ & NS \\
Atelectasis & $33(4 / 12)$ & $20(1 / 5)$ & $50(3 / 6)$ & NS \\
Cavities & $30(3 / 10)$ & $0(0 / 5)$ & $60(3 / 5)$ & NS \\
Pleural thickening & $0(0 / 8)$ & $0(0 / 6)$ & $0(0 / 2)$ & \\
\hline
\end{tabular}

Data are presented as per cent and, in parentheses, the numbers of improvement cases/numbers of available cases. The p-values shown are for the nonconverted versus converted group. NS: nonsignificant. nonconverted group have active, advanced disease. Although it is not clear when therapy should be started, the present findings suggest that early treatment may contribute to a cure of NTM.

It is clear that this retrospective study is bound by several limitations. Although all the patients in the present study were treated with a CAM-containing regimen, treatment dose was individually tailored. Two major differences which did not seem to follow ATS criteria in daily practice were recognised. The first was a lower dose of CAM and the second was the choice of rifampicin instead of rifabutin. In several studies, a high dose of CAM was significantly more effective than lowdose CAM with other companion drugs. However, at present, high-dose CAM $\left(1,000 \mathrm{mg} \cdot\right.$ day $\left.^{-1}\right)$ has not been approved for clinical use by the Ministry of Health and Welfare in Japan. A controlled trial will be necessary to determine the appropriate dose of CAM. Although the difference was not significant, the rate of successful treatment was $22(85 \%)$ out of 26 in the rifabutin-containing regimen, and $10(77 \%)$ out of 13 in the rifampicin regimen in the study by WALLACE JR et al. [4]. However, the disadvantage of rifabutin is that it causes adverse events, including uveitis and leukopaenia, which are infrequent with rifampicin. Furthermore, rifabutin is not currently available in Japan. It was not possible to determine whether alternative treatment regimens might have had a differential effect on outcome. The present retrospective study contains selection bias, as some patients were only followed by chest radiogram and not HRCT. In addition, the proportion of patients excluded from the present study is relatively high. In order to eliminate those biases and to understand the longitudinal course of HRCT findings, a prospective study will be needed. Since the current authors' institutions (Tenryu Hospital and Seirei Mikatahara Hospital) are regional referral centres for mycobacterial diseases, the extent of the CT scan abnormalities reported here may differ substantially from those found in the community. Referral bias might have increased the proportion of patients with more severe disease. Finally, a variety of previous CT techniques have been superseded by new developments [19]. In the future, the use of higher resolution images by reducing the slice thickness or applying a higher spatial resolution reconstruction algorithm technique may allow the better understanding of the outcome of MAC pulmonary disease.

In conclusion, high-resolution computed tomography may eventually play a role in the clinical evaluation and follow-up of patients with Mycobacterium avium complex pulmonary disease. A prospective study, with lower drop-out rate and more complete data may provide stronger evidence of prognostic implication of high-resolution computed tomography in the clinical evaluation and follow-up of patients with Mycobacterium avium complex pulmonary disease.

\section{ACKNOWLEDGEMENTS}

The authors thank T. Tsuchiya (Shizuoka City Shimizu Hospital, Shizuoka, Japan) and T. Naito (Hamamatsu University School of Medicine, Shizuoka, Japan) for helpful comments on statistical analysis.

\section{REFERENCES}

1 Henry MT, Inamdar L, O'Riordain D, Schweiger M, Watson JP. Nontuberculous mycobacteria in non-HIV patients: epidemiology, treatment and response. Eur Respir J 2004; 23: 741-746.

2 Tanaka E, Kimoto T, Tsuyuguchi K, et al. Effect of clarithromycin regimen for Mycobacterium avium complex pulmonary disease. Am J Respir Crit Care Med 1999; 160: 866-872.

3 Kobashi Y, Matsushima T. The effect of combined therapy according to the guidelines for the treatment of Mycobacterium avium complex pulmonary disease. Intern Med 2003; 42: 670-675.

4 Wallace RJ Jr, Brown BA, Griffith DE, Girard WM, Murphy DT. Clarithromycin regimens for pulmonary Mycobacterium avium complex. The first 50 patients. Am J Respir Crit Care Med 1996; 153: 1766-1772.

5 Dautzenberg B, Piperno D, Diot P, Truffot-Pernot C, Chauvin JP. Clarithromycin in the treatment of Mycobacterium avium lung infections in patients without AIDS. Clarithromycin Study Group of France. Chest 1995; 107: 1035-1040.

6 Moore EH. Atypical mycobacterial infection in the lung: CT appearance. Radiology 1993; 187: 777-782.

7 Swensen SJ, Hartman TE, Williams DE. Computed tomographic diagnosis of Mycobacterium avium-intracellulare complex in patients with bronchiectasis. Chest 1994; 105: 49-52.

8 Diagnosis and treatment of disease caused by nontuberculous mycobacteria. This official statement of the American Thoracic Society was approved by the Board of Directors, March 1997. Medical Section of the American Lung Association. Am J Respir Crit Care Med 1997; 156: S1-S25.

9 Hartman TE, Swensen SJ, Williams DE. Mycobacterium avium-intracellulare complex: evaluation with CT. Radiology 1993; 187: 23-26.

10 Kim JS, Tanaka N, Newell JD, et al. Nontuberculous mycobacterial infection: CT scan findings, genotype, and treatment responsiveness. Chest 2005; 128: 3863-3869. 
11 Kim TS, Koh WJ, Han J, et al. Hypothesis on the evolution of cavitary lesions in nontuberculous mycobacterial pulmonary infection: thin-section $\mathrm{CT}$ and histopathologic correlation. AJR Am J Roentgenol 2005; 184: 1247-1252.

12 Primack SL, Logan PM, Hartman TE, Lee KS, Muller NL. Pulmonary tuberculosis and Mycobacterium avium-intracellulare: a comparison of CT findings. Radiology 1995; 194 : 413-417.

13 Huang JH, Kao PN, Adi V, Ruoss SJ. Mycobacterium aviumintracellulare pulmonary infection in HIV-negative patients without preexisting lung disease: diagnostic and management limitations. Chest 1999; 115: 1033-1040.

14 Kubo K, Yamazaki Y, Hachiya T, et al. Mycobacterium avium-intracellulare pulmonary infection in patients without known predisposing lung disease. Lung 1998; 176: 381-391.

15 Tanaka D, Niwatsukino H, Oyama T, Nakajo M. Progressing features of atypical mycobacterial infection in the lung on conventional and high resolution CT (HRCT) images. Radiat Med 2001; 19: 237-245.

16 Fujita J, Ohtsuki Y, Suemitsu I, et al. Pathological and radiological changes in resected lung specimens in Mycobacterium avium intracellulare complex disease. Eur Respir J 1999; 13: 535-540.

17 Koh WJ, Lee KS, Kwon OJ, Jeong YJ, Kwak SH, Kim TS. Bilateral bronchiectasis and bronchiolitis at thin-section CT: diagnostic implications in nontuberculous mycobacterial pulmonary infection. Radiology 2005; 235: 282-288.

18 Obayashi Y, Fujita J, Suemitsu I, Kamei T, Nii M, Takahara J. Successive follow-up of chest computed tomography in patients with Mycobacterium avium-intracellulare complex. Respir Med 1999; 93: 11-15.

19 Fischbach F, Knollmann F, Griesshaber V, Freund T, Akkol E, Felix R. Detection of pulmonary nodules by multislice computed tomography: improved detection rate with reduced slice thickness. Eur Radiol 2003; 13: 2378-2383. 